\title{
Association between kidney stones and risk of developing stroke: a meta-analysis
}

\author{
Min Yuan ${ }^{1}$ (D) Huang-Yan Zhou ${ }^{2} \cdot$ Fan Hu$^{1} \cdot$ Shi-Ying Liu ${ }^{1} \cdot$ Wei Rao ${ }^{1} \cdot$ Ling-Feng Wu $^{1} \cdot$ Hong-Bing Nie $^{1}$. \\ Wen-Feng Cao ${ }^{1}$
}

Received: 12 August 2020 / Accepted: 2 February 2021 / Published online: 19 February 2021

(C) The Author(s) 2021

\begin{abstract}
Background Many studies have described the relationship between kidney stones and stroke, but the results are controversial, so we conducted this meta-analysis to estimate the relationship between kidney stones and the risk of developing stroke.

Methods Studies were marked with a comprehensive search of PubMed, EMBASE, Google, and ISI Web of Science databases through 25 March 2020. Hazard ratios (HRs) and 95\% confidence intervals (CIs) were extracted, and a random-effects model or fix-effects model was used to compute the pooled combined risk estimate. Heterogeneity was reported as $I^{2}$. We performed subgroup and sensitivity analysis to assess potential sources of heterogeneity.

Results Eight studies of seven articles involving 3,526,808 participants were included in the meta-analysis. Overall, kidney stones were associated with a moderate risk of stroke incidence (HR, $\left.1.24 ; 95 \% \mathrm{CI}, 1.11-1.40 ; I^{2}=79.6 \% ; p=0.000\right)$. We conducted a sensitivity analysis by removing the studies that had a high risk of bias. Heterogeneity subsequently decreased significantly, while an increased risk of stroke in patient with kidney stones was again demonstrated (HR, 1.16; 95\% CI, 1.11$\left.1.23 ; I^{2}=28.7 \% ; p=0.000\right)$. Stratifying analysis showed that the results were more pronounced for ischemic stroke (HR, $1.14 ; 95 \%$ CI, $\left.1.08-1.22 ; I^{2}=15.6 \% ; p=0.00\right)$ and the follow-up duration $\geq 10$ years $\left(\mathrm{HR}, 1.18 ; 95 \% \mathrm{CI}, 1.10-1.27 ; I^{2}=31.6 \% ; p=0.003\right)$.

Conclusions Our meta-analysis suggests that patients with kidney stones may have a modestly increased risk of developing stroke, especially in ischemic stroke. More large-scaled and clinical trials should be done to identify the relative impact of kidney stones on stroke outcomes in the future.
\end{abstract}

Keywords Kidney stones $\cdot$ Stroke $\cdot$ Cerebrovascular disease $\cdot$ Meta-analysis

\section{Introduction}

Stroke is a common cerebrovascular disease known for its high incidence, high mortality, and increased disability rate.

Min Yuan

yuanmin201314@sina.com

Wen-Feng Cao

caowf-2004@126.com

Huang-Yan Zhou

zhouhuangyan163@163.com

$\mathrm{Fan} \mathrm{Hu}$

hudaming2005@126.com

Shi-Ying Liu

shiying_liu@hotmail.com

Wei Rao

raowei_991384@sina.com
Depending on statistics, it is the second-largest cause of death and disability in the world [1-3]. On average, someone died of a stroke every $4 \mathrm{~min}$ [4]. Studies showed that about 780,000 Americans experience a new or recurrent stroke every year

Ling-Feng $\mathrm{Wu}$

wusky2000@126.com

Hong-Bing Nie

Mrniehongbing@163.com

1 Department of Neurology, Jiangxi Provincial People's Hospital Affiliated to Nanchang University, No. 152, Aiguo Road, Nanchang 330006, Jiangxi, China

2 Department of Blood Transfusion, Jiangxi Cancer Hospital, Nanchang 330029, Jiangxi, China 
[5]. In 2005, accounted for 5.7 million deaths, 16 million firsttime stroke events worldwide, and it is speculated that these numbers may reach 7.8 million and 23 million by 2030 [5], respectively, according to estimates by the WHO, which creates a significant public health burden on the society. Therefore, identifying primary prevention for stroke risk and any possible means to prevent a stroke should be a critical public health priority, especially among young adults.

Kidney stones are a relatively prevalent disease in our daily life, especially in Western civilizations [6, 7]. Many studies have assessed the association of kidney stones with stroke risk [8-13]. However, the role of kidney stones in stroke is still controversial. A previous meta-analysis showed that kidney stones are associated with increased cardiovascular risk, but only three stroke studies were included [14]. Simultaneously, they did not analyze the influence of study design, stroke type, geographic area, follow-up time, and study quality. The results are also quite heterogeneous. Besides, Peng et al. [15] conducted a meta-analysis only in 4 studies, and the description of the subgroup analysis is inconsistent with that of Fig. 3. What is more, the data of meta-analysis was just limited to May 2016. Furthermore, we found several articles that they did not include $[9,13,16]$. Recent studies involving the relationship between kidney stones and risk of stroke incidence were published from then on [12]. To obtain a more comprehensive estimate of kidney stones' putative influence on stroke, we conducted a meta-analysis of all related studies to determine the association between kidney stones and stroke risk.

\section{Materials and methods}

\section{Literature search}

Our meta-analysis was conducted and reported according to the recommendations of Preferred Reporting Items for Systematic Reviews and Meta-Analyses (PRISMA) Statement [17]. We searched PubMed, EMBASE, Google, and ISI Web of Science databases for the related published articles through 25 March 2020. We used the following keywords: "kidney stones," "renal stones," "renal calculus," "kidney calculi," "nephrolith," "nephrolithiasis," "stroke," "cerebrovascular disorders," "cerebrovascular accidents," "cerebrovascular disease," "cerebral infarct," "ischemic stroke," and "intracranial hemorrhage." There were no any language restrictions.

\section{Study selection}

Studies were included for our meta-analysis if they fulfilled the following criteria: (1) the study had a cohort design, case control, or cross-sectional design; (2) assignment of kidney stones as the baseline exposure, stroke was the outcome measure; and (3) the reported quantitative estimates of the multivariate-adjusted hazard ratios (HRs), odds ratio (OR) or relative risk (RR), and 95\% confidence intervals (CIs) for stroke associated with kidney stones. The original effect size value (OR, RR) was used 1:1 as input for HR in the sense of best estimation.

\section{Data abstraction and quality assessment}

All data were independently abstracted in duplicate using a standard data collection form. When necessary, the original authors were contacted for supplementary information. Discrepancies were settled by consensus. The following data were extracted from each study: first author's last name, year of publication, study design, source of population, the country where the analysis was performed, size of the study, age range or average age, follow-up time, assessment of kidney stones and stroke, and study quality. The Newcastle-Ottawa Scale (NOS) was utilized to assess the quality of the studies [18]. The full score was 9 stars. If the score of studies met $\geq 7$ awarded stars, it means high-quality studies.

\section{Statistical analysis}

Stata 12 (Stata Corporation, College Station, TX) was utilized to perform the meta-analysis. $P$ values were 2 -sided, and $p<$ 0.05 was considered statistically significant. For each study, the HR was used for the measurement data and presented with $95 \%$ confidence intervals. The chi-square test's degree of heterogeneity among the results was estimated (the $p$ value $<0.1$ was considered significant). Whenever significant heterogeneity ( $p$ value $<0.10$ or $I^{2}$ score $>50 \%$ ) was achieved, the random-effects model was used. If no significant heterogeneity across studies was found, a fixed-effect model was selected to pool the data [19]. The pStatemenias was identified with the symmetry of the funnel plot, Egger's test, and Begg's test. Sensitivity analyses and subgroups were performed where appropriate.

\section{Results}

\section{Literature search}

A flow chart displaying the results of the literature search and selection is presented in Fig. 1. We initially identified 879 potential publications through the systematic retrieval of PubMed, EMBASE, Google, and ISI Web of Science databases. After excluding duplicate records and studies or after scanning the title and abstract that did not fulfill our inclusion criteria, 19 studies remained. We evaluated the full texts of these 19 publications and excluded 12 studies for the 
Records identified through database search $(\mathrm{n}=879)$

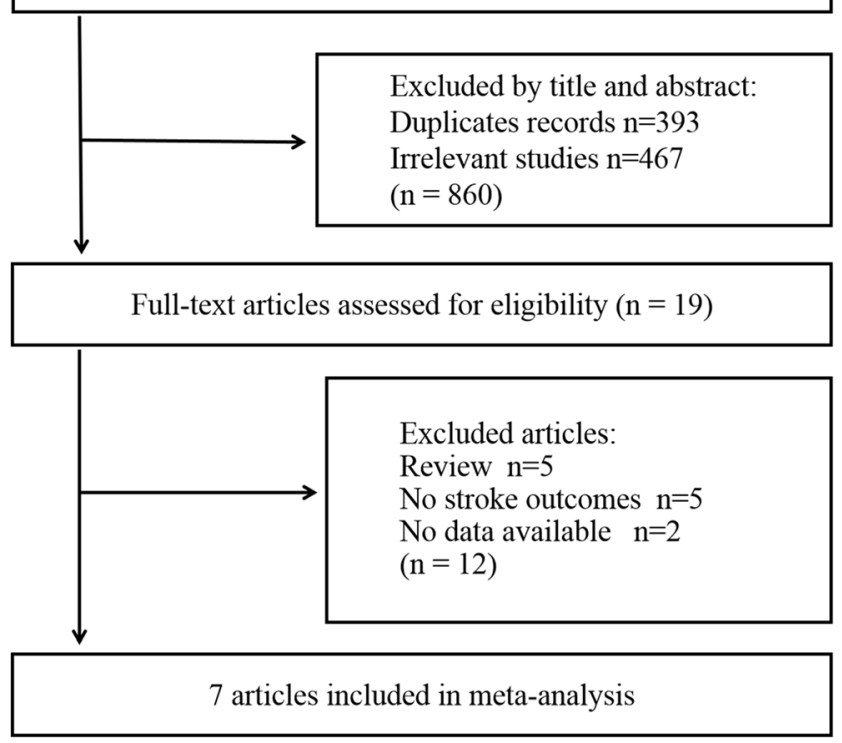

Fig. 1 Flow chart of study selection

following reasons: reviews ( $n=5$ ) [18-24], no stroke outcomes $(n=5)$ [25-29], and no data available $(n=2)$ [30, 31]. Seven articles in eight studies finally met the inclusion criteria and were included in the meta-analysis $[8-13,16]$.

\section{Study characteristics}

The characteristics of the studies and their participants are presented in Table 1. Seven articles from eight studies involving 3,526,808 participants were included in the meta-analysis. Of these seven articles $[8-13,16]$, there are two research results in each of two articles $[10,12]$. Alexander et al. [10] elaborated on cardiovascular events in the primary and laboratory cohorts. Kim et al. [12] described the cohort studies of ischemic stroke and hemorrhagic stroke, and, respectively, all have available data. However, the "laboratory cohort" is not an independent group of participants; it is a mere subset from the "primary cohort," so the data were not included in the analysis. Three studies were conducted in Asia [8, 9, 12]; three studies were conducted in Europe $[11,13,16]$, and one in a North American country (Canada) [10]. Six articles are cohort studies [8-13], and one is a cross-sectional study [16]. Only three of the seven articles identified ischemic stroke $[8,12,13]$, and one pointed to hemorrhagic stroke [12], and data were available. All studies included both men and women, but no specific operational data. The duration of follow-up varied from 1.1 to 12 years, with an average of 7.6 years. The assessment of kidney stones varied across studies. In most studies, kidney stones were measured using International Classification of Diseases-9-CM (ICD-9-CM) [12] and ICD-10 [12]; three studies were measured using a self-reported questionnaire as well as clinical examinations $[11,13,16]$. In most studies, stroke was evaluated by medical records based on ICD-9 or ICD-10. Only one study was measured using self-reported questionnaire [16]. All studies provided risk estimates adjusted for related covariance; the quality score of studies ranged from 6 to 9 , and the average score was 7.7. Among them, there were five studies $[8-10,12,13]$ with scores $\geq 7$.

\section{Kidney stones and stroke incidence}

Eight studies of seven articles [8-13, 16] elaborated the relationship between kidney stones and stroke. We proceeded to a meta-analysis of all the data. From the results of the analysis, there was statistically significant heterogeneity in our study $\left(I^{2}=79.6 \%\right)$, so we used the random-effects model. Multivariable-adjusted HR of stroke incidence concerning kidney stone from individual studies is presented in Fig. 2. Participants with kidney stones experienced a moderately increased risk for stroke development based on eight studies compared with non-kidney stone (HR, 1.24; 95\% CI, 1.11$1.40 ; p=0.000)$. When we excluded the cross-sectional study [16] and analyzed all cohort studies, the combined HR was $1.24 ; 95 \%$ CI, $1.09-1.40 ; p=0.001$. The result was statistically significant, confirming the increased risk of stroke in patients with kidney stones again.

\section{Stratifying analysis}

There was statistically significant heterogeneity in the overall analysis, and the source of population, duration of follow-up, study design, and methodological quality varied across studies. Therefore, we also conducted subgroup analyses further to elucidate the effect of kidney stones on stroke risk. Table 2 showed pooled HR for stroke stratified by study design, geographical area, stroke type, duration of follow-up, and study quality. The HR for Asian and North American studies indicated that kidney stones were associated with an increased risk of stroke (Asian: 1.24 (95\% CI, 1.04-1.48; $p=0.014$; North American: 1.26 (95\% CI, 1.12-1.42; $p=0.000$ ), while Europe studies were not associated with a risk of stroke (HR, 1.24 (95\% CI, 0.93-1.64; $p=0.141$ ). The pooled estimate of multivariate HRs based on three studies $[8,12,13]$ was $1.14(95 \%$ CI, 1.08-1.22; $p=0.000$ ) among ischemic stroke and 1.07 among hemorrhagic stroke (95\% CI, 0.91-1.26; $p=0.415$ ) based on one study [12]. These results suggest that kidney stones are associated with the risk of ischemic stroke. Besides, increases in stroke events were also found in the subgroup meta-analysis of study quality $(\geq 7$ or $<7$ ) and duration of follow-up ( $\geq 10$ years), which indicated that kidney stones are closely associated with an increased risk of stroke regardless of study quality. 


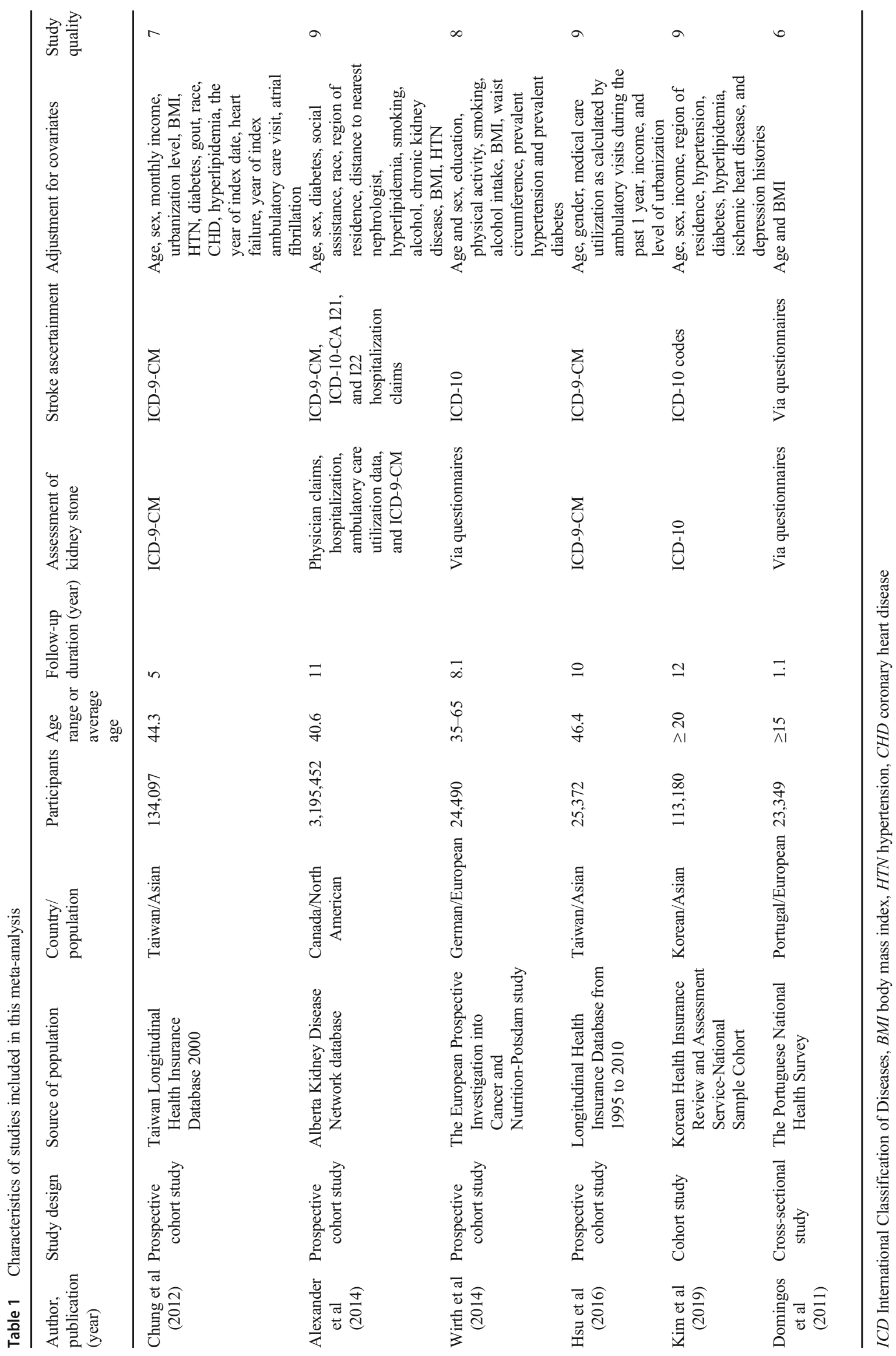


Fig. 2 Random-effects analysis of fully adjusted studies for the association between kidney stones and stroke risk

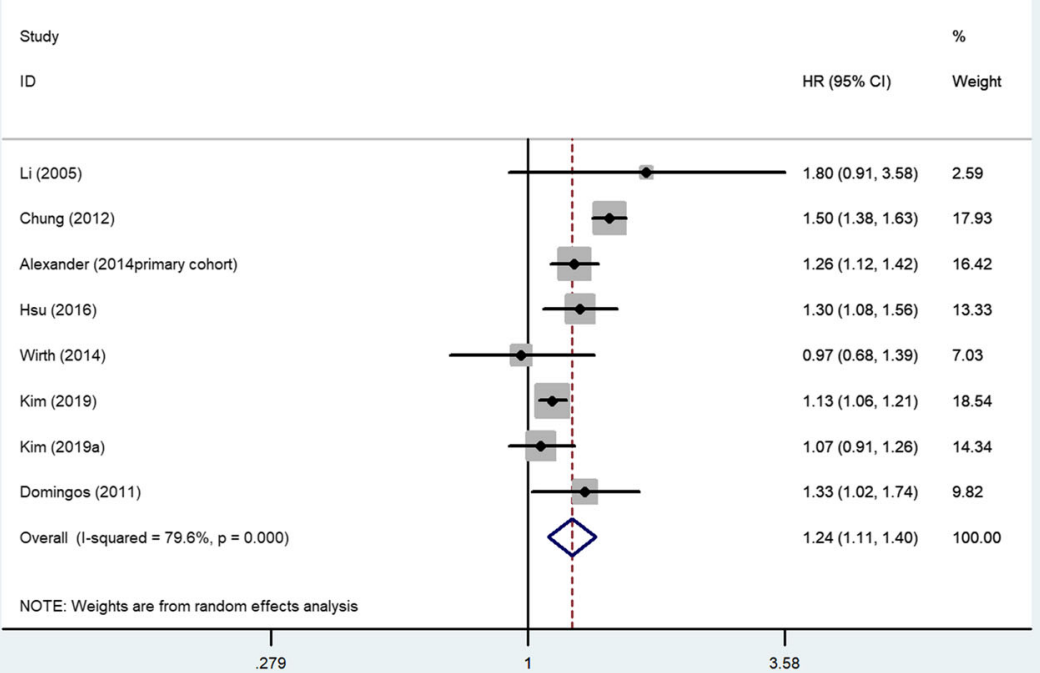

(1)

\section{Sensitivity analysis and publication bias}

We tested the robustness of our results in a sensitivity analysis by omitting one study at one time. The results showed that the study of Chung et al. [9] substantially affected the pooled HRs. When we deleted the study and calculated the pooled HRs with the fixed-effect model for the remaining of the studies, significant increases in stroke events were also found: HR, 1.16 (95\% CI, 1.11-1.23; $p=0.000$ ), the heterogeneity was significantly decreased $\left(I^{2}=28.7 \%, p=0.209\right)$ (Fig. 3). There was no evidence of publication bias by inspection of the funnel plot (Fig. 4). Further study showed no evidence of substantial publication bias was observed among studies for stroke risk from the Begg's test $(p=0.322)$ and Egger's test $(p=0.929)$.

\section{Discussion}

Our meta-analysis from eight studies of seven articles confirmed a positive association between kidney stones and the stroke risk after adjusting established cardiovascular risk factors, with an overall 1.24-fold increased risk compared with those without a history of kidney stones.

Over the past decades, despite extensive studies investigating the kidney stone's role on either cardiovascular disease or stroke, it remains unclear whether the association between kidney stones and risk of stroke incidence is causal. Some of the studies suggested that kidney stones were associated with an increased risk of stroke, and the others failed to find the association. Many studies showed that kidney stones are associated with coronary heart disease (CHD) [10, 32], hypertension [33], diabetes [34], atherosclerosis [28], and metabolic

Table 2 Stratified analyses of kidney stones and stroke incidence

\begin{tabular}{|c|c|c|c|c|c|c|c|c|}
\hline \multirow[b]{2}{*}{ Subgroup } & \multirow[b]{2}{*}{ Categories } & \multirow[b]{2}{*}{ No. of cohorts } & \multirow[b]{2}{*}{ Adjusted HR } & \multirow[b]{2}{*}{$95 \% \mathrm{Cl}$} & \multicolumn{3}{|c|}{ Heterogeneity test } & \multirow[b]{2}{*}{$p$ value of pooled effect } \\
\hline & & & & & $X^{2}$ & $p$ value & $I^{2}, \%$ & \\
\hline \multirow[t]{2}{*}{ Study design } & Cohort study & 7 & 1.24 & $1.09-1.40$ & 34.05 & 0.000 & 82.4 & 0.001 \\
\hline & Cross-sectional study & 1 & 1.33 & $1.02-1.74$ & 0.00 & . & . & 0.036 \\
\hline \multirow[t]{3}{*}{ Geographical area } & Asian & 4 & 1.24 & $1.04-1.48$ & 31.03 & 0.000 & 90.3 & 0.014 \\
\hline & Europe & 3 & 1.24 & $0.93-1.64$ & 3.21 & 0.201 & 37.7 & 0.141 \\
\hline & North America & 1 & 1.26 & $1.12-1.42$ & 0.00 & . & . & 0.000 \\
\hline \multirow[t]{2}{*}{ Stroke type } & Ischemic stroke & 3 & 1.14 & $1.08-1.22$ & 2.37 & 0.306 & 15.6 & 0.000 \\
\hline & Hemorrhagic stroke & 1 & 1.07 & $0.91-1.26$ & 0.00 & . & . & 0.415 \\
\hline \multirow[t]{2}{*}{ Quality score } & $<7$ & 2 & 1.38 & $1.08-1.78$ & 0.65 & 0.420 & 0.0 & 0.010 \\
\hline & $\geq 7$ & 6 & 1.22 & $1.07-1.39$ & 32.93 & 0.000 & 84.8 & 0.003 \\
\hline \multirow[t]{2}{*}{ Follow-up duration (year) } & $<10$ & 3 & 1.34 & $0.97-1.86$ & 5.76 & 0.056 & 65.3 & 0.079 \\
\hline & $\geq 10$ & 5 & 1.18 & $1.10-1.27$ & 5.85 & 0.211 & 31.6 & 0.003 \\
\hline
\end{tabular}


Fig. 3 Fixed-effects analysis of fully adjusted results for the association between kidney stones and stroke risk after sensitivity analysis

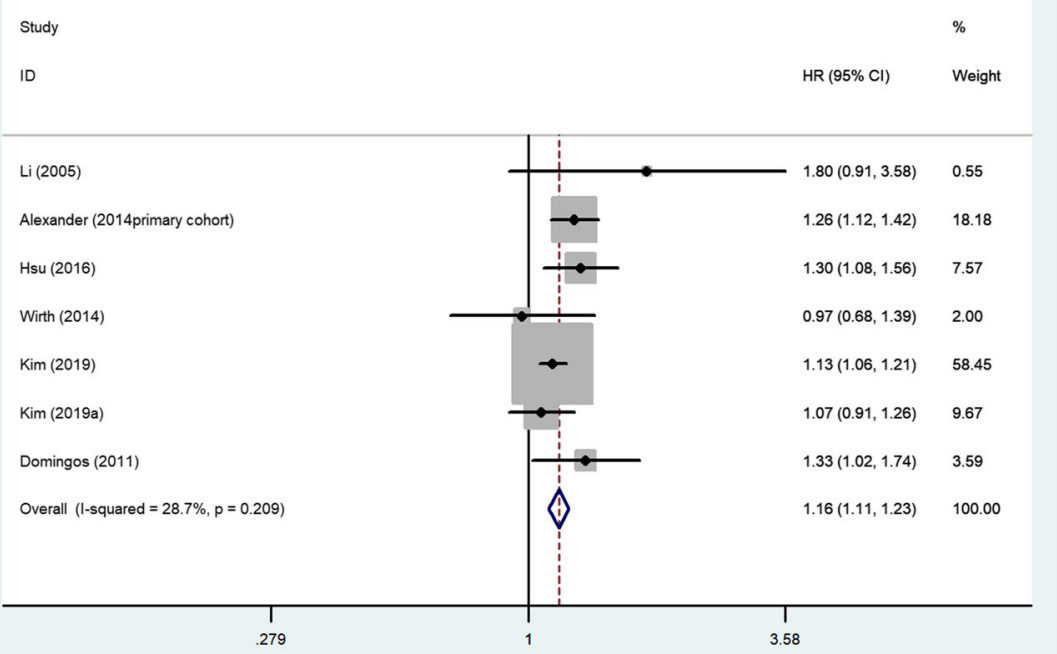

syndrome [35], which are the risk factor for CHD and stroke; thus, patients with nephrolithiasis might have a high stroke risk. Through a 10-year follow-up of people in Taiwan, Hsu et al. [8] found a positive correlation between the increased risk of kidney stones and stroke, which is consistent with previous epidemiological evidence $[9,10,16,31]$ and the results of our current study. Lin et al. [30] analyzed data from a large number of patients with nephrolithiasis. They matched controls from a national insurance claim dataset of 22 million enrollees in Taiwan with 13 years of follow-up. They found that nephrolithiasis is associated with an increased risk of ischemic stroke, particularly for women and the younger population, based on the population-based study. This is consistent with our study's subgroup analysis showing that kidney stones are associated with the risk of ischemic stroke, but there is no significant correlation for hemorrhagic stroke. However, because this article does not provide useful data after adjusting the established risk factors, we omit this article in the study.

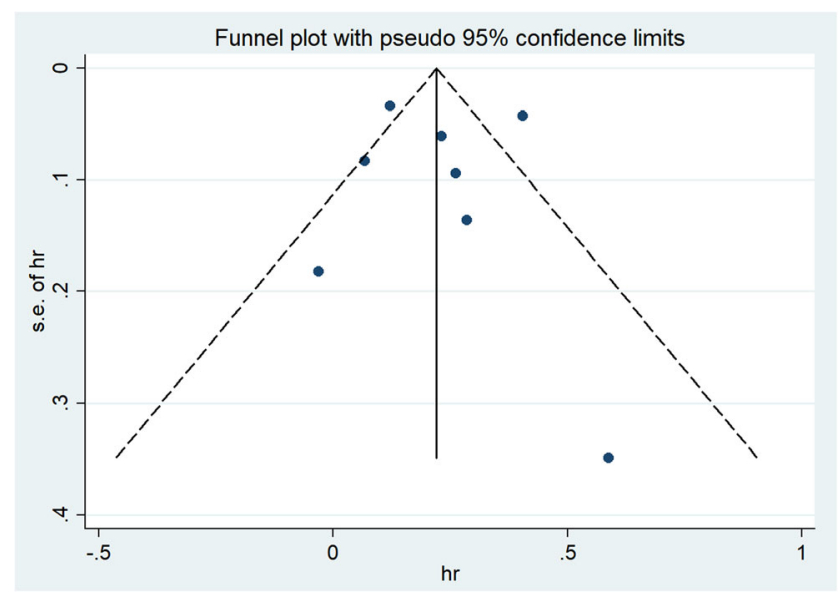

Fig. 4 Funnel plots of kidney stones and the risk of stroke incidence
However, Li et al. [11] and Wirth et al. [13] reported that patients with renal calculus did not associate with stroke incidence. Moreover, the laboratory cohort and primary cohort studies conducted by Alexander et al. [10], including 3,195,452 participants, showed two different results. Meanwhile, Kim et al. [12] found differences in ischemic stroke incidence and hemorrhagic stroke in patients with kidney stones in a cohort of 113,180 participants in 2019. Therefore, we carried out a meta-analysis of all related studies to quantitatively identify whether kidney stones are associated with the risk of stroke and eliminate these disputes as much as possible, guiding the primary prevention of stroke patients in the clinic.

For the moment, the mechanisms underlying the increase of consequent stroke events in kidney stones patients are not well understood. Previous studies showed that kidney stones are associated with hypertension, smoking, obesity, diabetes, and hyperlipidemia, which are all known risk factors for stroke [33, 34, 36], and kidney stone formers had increased evidence of subclinical atherosclerosis with the pathogenesis of stroke event [28]. Other studies have shown that obesity and insulin resistance can lead to poor ammoniation, so diabetes can increase the risk of uric acid kidney stones by inducing low urine $\mathrm{pH}$ [37]. Furthermore, lots of kidney stones are composed of calcium. Hypercalciuria should be the most significant risk factor for the development of kidney stones [26, 38]. In contrast, patients with increased calcium precipitation in parts of the body, such as the intracranial region and coronary vessels, may link the underlying pathophysiology of the formation of calcium precipitations in the renal tubule, which would, in turn, cause the clinical sequelae of stroke [39].

However, heterogeneity between studies was found in kidney stones and risk of stroke incidence. Therefore, we chose the random-effects model to analyze the results; stratifying analysis and sensitivity analysis were also used to reduce 
heterogeneity and find the source of heterogeneity. Within the stratifying analysis, we examined study design, geographical area, stroke type, length of follow-up, and study quality as possible sources of heterogeneity, and some studies showed that these did not show any significant heterogeneity between studies. Although the stratifying analysis could not explain the heterogeneity level in interpreting the results, several studies' differences are worth discussing. When we conducted a sensitivity analysis by omitting one study at one time, the results showed that the study of Chung et al. [9] substantially affected the pooled HRs. When we deleted the study and calculated the pooled HRs for the remaining of the studies, the heterogeneity was significantly decreased (HR, 1.16; 95\% CI, 1.11-1.23; $I^{2}=28.7 \% ; p=0.000$ ), we found that the study was a prospective cohort study, which was conducted by the source from the Taiwan Longitudinal Health Insurance Database 2000. The diagnosis of kidney stone and stroke in this article depends on the doctors' and hospitals' data, rather than based on standardized criteria, and residual confounding factors and surveillance biases also existed. As we all know, according to the GRADE system, five factors may lead to a decrease in the quality of evidence, including the risk of bias, inconsistent results, indirectness of evidence, inaccuracy of the results, and publication bias. In this article, we believe that the inconsistency in rating the quality of evidence is the main reason for the current results and heterogeneity. Of course, differences in environmental factors, countries, methodological factors in study design, and how the studies were conducted should attribute to the observed heterogeneity. Therefore, the existence of heterogeneity requires a proper and careful understanding of the current meta-analysis findings.

This study has several important strengths compared with previous meta-analyses. To our knowledge, this article provides a more systematic explanation of the relationship between kidney stones and stroke risk. It also conducts stratifying analysis on some known influencing factors. At the same time, sensitivity analysis is used to screen the research results' heterogeneity. Furthermore, the Newcastle-Ottawa Scale was used to assess individual studies' quality. Most of them were of high quality. Besides, we use the funnel chart to directly show that there is no publication bias in the research results, which are further confirmed by Begg's and Egger's tests. Therefore, the results should be reliable.

There were also many limitations to our meta-analysis. Firstly, a limited number of studies were included, although we strove to find all related studies. Only eight studies of seven articles were included in our meta-analysis. Secondly, although we considered a multitude of risk factors in the multivariable analysis, the possibility of residual confounding or confounding by unmeasured factors, which cannot be ruled out in any observational study, must be acknowledged. Thirdly, although we used the random-effects model and stratifying analysis, the results still have some heterogeneity. Also, the number of studies included in the subgroup was small, lacking sufficient reliability to confirm a relationship in a definitive manner. The number of available studies of different stroke outcomes and differences in study design, country, and assessment method included in this meta-analysis was moderate. Therefore, the results could be influenced by factors like methodological differences, regional differences, etc.

\section{Conclusion}

Our meta-analysis found a moderate association between kidney stones and the risk of stroke incidence after adjustment of established cardiovascular risk factors, especially in ischemic stroke. To efficiently assess the association and causality of kidney stones on stroke, more large-scaled and clinical trials should be done to identify the relative impact of kidney stones on stroke outcomes and explore whether effective treatment of kidney stones may prevent or improve the course of stroke.

Supplementary Information The online version contains supplementary material available at https://doi.org/10.1007/s10072-021-05113-5.

Acknowledgements MY and WFC conceived and designed the experiments. WFC, FH, SYL, WR, LFW, MY, and HYZ analyzed the data. MY wrote the paper. FH, SYL, WR, LFW, HBN, MY, and HYZ performed the literature search and the data extraction. All authors saw and approved the final version of the manuscript. We thank the editors of the journal for editing the manuscript.

Funding The present study was funded by the scientific research project of the traditional Chinese Medicine of Jiangxi Provincial Health and Family Planning Commission (No. 2018A116). The authors thank the participants of this study for their important contributions.

Data availability All primary studies surveyed are published. To the best of our knowledge, no unpublished studies were available.

\section{Declarations}

Ethical approval None.

Conflict of interest The authors declare that they have no competing interests.

Open Access This article is licensed under a Creative Commons Attribution 4.0 International License, which permits use, sharing, adaptation, distribution and reproduction in any medium or format, as long as you give appropriate credit to the original author(s) and the source, provide a link to the Creative Commons licence, and indicate if changes were made. The images or other third party material in this article are included in the article's Creative Commons licence, unless indicated otherwise in a credit line to the material. If material is not included in the article's Creative Commons licence and your intended use is not permitted by statutory regulation or exceeds the permitted use, you will need to obtain permission directly from the copyright holder. To view a copy of this licence, visit http://creativecommons.org/licenses/by/4.0/. 


\section{References}

1. Mozaffarian D, Benjamin EJ, Go AS, Arnett DK, Blaha MJ, Cushman M, Das SR, de Ferranti S, Despres JP, Fullerton HJ, Howard VJ, Huffman MD, Isasi CR, Jimenez MC, Judd SE, Kissela BM, Lichtman JH, Lisabeth LD, Liu S, Mackey RH, Magid DJ, McGuire DK, Mohler ER, Moy CS, Muntner P, Mussolino ME, Nasir K, Neumar RW, Nichol G, Palaniappan L, Pandey DK, Reeves MJ, Rodriguez CJ, Rosamond W, Sorlie PD, Stein J, Towfighi A, Turan TN, Virani SS, Woo D, Yeh RW, Turner MB (2016) Heart disease and stroke Statistics-2016 update: a report from the American Heart Association. Circulation 133: e38-e360

2. O'Donnell MJ, Chin SL, Rangarajan S, Xavier D, Liu L, Zhang H, Rao-Melacini P, Zhang X, Pais P, Agapay S, Lopez-Jaramillo P, Damasceno A, Langhorne P, McQueen MJ, Rosengren A, Dehghan M, Hankey GJ, Dans AL, Elsayed A, Avezum A, Mondo C, Diener HC, Ryglewicz D, Czlonkowska A, Pogosova N, Weimar C, Iqbal R, Diaz R, Yusoff K, Yusufali A, Oguz A, Wang X, Penaherrera E, Lanas F, Ogah OS, Ogunniyi A, Iversen HK, Malaga G, Rumboldt Z, Oveisgharan S, Al HF, Magazi D, Nilanont Y, Ferguson J, Pare G, Yusuf S (2016) Global and regional effects of potentially modifiable risk factors associated with acute stroke in 32 countries (INTERSTROKE): a case-control study. Lancet 388:761-775

3. Zambrano MD, Miller EC (2019) Maternal stroke: an update. Curr Atheroscler Rep 21:33

4. Strong K, Mathers C, Bonita R (2007) Preventing stroke: saving lives around the world. Lancet Neurol 6:182-187

5. Sidney S, Rosamond WD, Howard VJ, Luepker RV (2013) The "heart disease and stroke statistics-2013 update" and the need for a national cardiovascular surveillance system. Circulation 127:21-23

6. Sakhaee K (2014) Epidemiology and clinical pathophysiology of uric acid kidney stones. J Nephrol 27:241-245

7. Rahman SH, Papadakis GZ, Keil MF, Faucz FR, Lodish MB, Stratakis CA (2016) Kidney stones as an Underrecognized clinical sign in pediatric Cushing disease. J Pediatr 170:273-277

8. Hsu CY, Chen YT, Huang PH, Leu HB, Su YW, Chiang CH, Chen JW, Chen TJ, Lin SJ, Chan WL (2016) The association between urinary calculi and increased risk of future cardiovascular events: a nationwide population-based study. J Cardiol 67:463-470

9. Chung SD, Liu SP, Keller JJ, Lin HC (2012) Urinary calculi and an increased risk of stroke: a population-based follow-up study. BJU Int 110:E1053-E1059

10. Alexander RT, Hemmelgarn BR, Wiebe N, Bello A, Samuel S, Klarenbach SW, Curhan GC, Tonelli M (2014) Kidney stones and cardiovascular events: a cohort study. Clin J Am Soc Nephrol 9:506-512

11. Li C, Engstrom G, Hedblad B, Berglund G, Janzon L (2005) Risk factors for stroke in subjects with Normal blood pressure: a prospective cohort study. Stroke 36:234-238

12. Kim SY, Song CM, Bang W, Lim J, Park B, Choi HG (2019) Nephrolithiasis predicts ischemic stroke: a longitudinal follow-up study using a national sample cohort. Int J Med Sci 16:1050-1056

13. Wirth J, Weikert S, di Giuseppe R, Fritsche A, Boeing H, Weikert C (2014) Relationship between renal calculi and the risk of myocardial infarction and stroke: results from the EPIC-Potsdam study. Clin Nephrol Urol Sci 3:2054-7161

14. Liu Y, Li S, Zeng Z, Wang J, Xie L, Li T, He Y, Qin X, Zhao J (2014) Kidney stones and cardiovascular risk: a meta-analysis of cohort studies. Am J Kidney Dis 64:402-410

15. Peng JP, Zheng H (2017) Kidney stones may increase the risk of coronary heart disease and stroke: a PRISMA-compliant meta-analysis. Medicine (Baltimore) 96:e7898
16. Domingos F, Serra A (2011) Nephrolithiasis is associated with an increased prevalence of cardiovascular disease. Nephrol Dial Transplant 26:864-868

17. Liberati A, Altman DG, Tetzlaff J, Mulrow C, Gotzsche PC, Ioannidis JP, Clarke M, Devereaux PJ, Kleijnen J, Moher D (2009) The PRISMA statement for reporting systematic reviews and meta-analyses of studies that evaluate health care interventions: explanation and elaboration. PLoS Med 6:e1000100

18. Stang A (2010) Critical evaluation of the Newcastle-Ottawa scale for the assessment of the quality of nonrandomized studies in metaanalyses. Eur J Epidemiol 25:603-605

19. DerSimonian R, Laird N (1986) Meta-analysis in clinical trials. Control Clin Trials 7:177-188

20. Marangella M (2016) Medical management of urinary calculi: up to date 2016. Urologia 83:110-123

21. Bolland MJ, Grey A, Reid IR (2013) Calcium supplements and cardiovascular risk: 5 years on. Ther Adv Drug Saf 4:199-210

22. Gambaro G, Ferraro PM, Capasso G (2012) Calcium nephrolithiasis, metabolic syndrome and the cardiovascular risk. Nephrol Dial Transplant 27:3008-3010

23. Lange JN, Mufarrij PW, Wood KD, Holmes RP, Assimos DG (2012) The association of cardiovascular disease and metabolic syndrome with nephrolithiasis. Curr Opin Urol 22:154-159

24. Ernandez T, Bonny O (2014) Kidney stone as a cardiovascular risk marker. Rev Med Suisse 10:1656-1660

25. Shu X, Cai H, Xiang Y, Li H, Lipworth L, Miller NL, Zheng W, Shu X, Hsi RS (2017) Nephrolithiasis among middle aged and elderly urban Chinese: a report from prospective cohort studies in Shanghai. J Endourol 31:1327-1334

26. Vezzoli G, Macrina L, Rubinacci A, Spotti D, Arcidiacono T (2016) Intestinal calcium absorption among Hypercalciuric patients with or without calcium kidney stones. Clin J Am Soc Nephrol 11: $1450-1455$

27. Glover LM, Bass MA, Carithers T, Loprinzi PD (2016) Association of kidney stones with atherosclerotic cardiovascular disease among adults in the United States: considerations by race-ethnicity. Physiol Behav 157:63-66

28. Reiner AP, Kahn A, Eisner BH, Pletcher MJ, Sadetsky N, Williams OD, Polak JF, Jacobs DJ, Stoller ML (2011) Kidney stones and subclinical atherosclerosis in young adults: the CARDIA study. J Urol 185:920-925

29. Ramey SL, Franke WD, Shelley MN (2004) Relationship among risk factors for nephrolithiasis, cardiovascular disease, and ethnicity: focus on a law enforcement cohort. AAOHN J 52:116-121

30. Lin SY, Lin CL, Chang YJ, Hsu WH, Lin CC, Wang IK, Chang CT, Chang CH, Lin MC, Kao CH (2016) Association between kidney stones and risk of stroke: a Nationwide population-based cohort study. Medicine (Baltimore) 95:e2847

31. Chou PS, Chang WP, Chou YH (2018) Urolithiasis is associated with an increased risk of stroke: a population-based 5-year followup study. Intern Med J 48:445-450

32. Ferraro PM, Taylor EN, Eisner BH, Gambaro G, Rimm EB, Mukamal KJ, Curhan GC (2013) History of kidney stones and the risk of coronary heart disease. JAMA 310:408-415

33. Obligado SH, Goldfarb DS (2008) The association of nephrolithiasis with hypertension and obesity: a review. Am J Hypertens 21:257-264

34. Taylor EN, Stampfer MJ, Curhan GC (2005) Diabetes mellitus and the risk of nephrolithiasis. Kidney Int 68:1230-1235

35. Inci M, Demirtas A, Sarli B, Akinsal E, Baydilli N (2012) Association between body mass index, lipid profiles, and types of urinary stones. Ren Fail 34:1140-1143

36. Jion YI, Raff A, Grosberg BM, Evans RW (2015) The risk and management of kidney stones from the use of topiramate and zonisamide in migraine and idiopathic intracranial hypertension. Headache 55:161-166 
37. Taylor EN, Stampfer MJ, Curhan GC (2005) Diabetes mellitus and the risk of nephrolithiasis. Kidney Int 68:1230-1235

38. Sethmann I, Wendt-Nordahl G, Knoll T, Enzmann F, Simon L, Kleebe HJ (2016) Microstructures of Randall's plaques and their interfaces with calcium oxalate monohydrate kidney stones reflect underlying mineral precipitation mechanisms. Urolithiasis

39. Malihi Z, Wu Z, Stewart AW, Lawes CM, Scragg R (2016) Hypercalcemia, hypercalciuria, and kidney stones in long-term studies of vitamin D supplementation: a systematic review and meta-analysis. Am J Clin Nutr 104:1039-1051

Publisher's note Springer Nature remains neutral with regard to jurisdictional claims in published maps and institutional affiliations. 